Page S-1

\title{
Determination of Klebsiella pneumoniae Susceptibility to Antibiotic Using Infrared Microscopy
}

Uraib Sharaha, ${ }^{\dagger}$ Manal Suleiman, ${ }^{\dagger}$ George Abu-Aqil, ${ }^{\dagger}$ Klaris Riesenberg, ${ }^{\ddagger}$ Itshak Lapidot, ${ }^{\S}$ Ahmad Salman*," and Mahmoud Huleihel*, ${ }^{\dagger}$

${ }^{\dagger}$ Department of Microbiology, Immunology and Genetics, Faculty of Health Sciences, Ben-Gurion University of the Negev, Beer-Sheva 84105, Israel.

${ }^{\ddagger}$ Soroka University Medical Center, Beer-Sheva 84105, Israel.

${ }^{\S}$ Department of Electrical and Electronics Engineering, ACLP-Afeka Center for Language Processing, Afeka Tel-Aviv Academic College of Engineering, TelAviv 69107, Israel.

" Department of Physics, SCE - Shamoon College of Engineering, Beer-Sheva 84100, Israel.

*Email: ahmad@sce.ac.il. Phone: +972-8-6475794.

*Email: mahmoudh@bgu.ac.il. Phone: +972-8-6479867.

U.S., M.S., and G.A.-A. contributed equally.

I.L., A.S., and M.H. contributed equally as PIs. 


\title{
Page S-2
}

\begin{abstract}
Figure $\mathrm{S} 1$ shows 12 measurements acquired from different sites of the same bacterial isolates. As can be seen from the figure, the spectra overlay each other, which indicates the high reproducibility of the measurements. The RF and XGBoost classifiers evaluated different combinations of Klebsiella pneumoniae and antibiotics (Figure 1); one combination or the other produced a slightly better result. In those cases, the classifier that did not produce the best results was identified as "second" classifier. The tables and figures in this document show the results produced after evaluation by the "second" classifier (except Figure S3); "best" classifier results are shown in the primary manuscript.
\end{abstract}

Figure S2 shows the spectra of Klebsiella pneumoniae that are resistant and sensitive to specific antibiotics. As can be seen from Figure S2, the spectra are similar and overlap.

Table S1 summarizes the optimal hyper-parameters for the second classifier. (We identified RF as the second classifier when XGBoost produced better results; we identified XGBoost as the second classifier when RF produced better results.)

Figure S3 presents information about cephalexin, ciprofloxacin, gentamicin, and sulfamethoxazole, in a format similar to Figure 3.

Figure S4 (for amoxicillin, ceftazidime, ceftriaxone, cefuroxime, and cefuroxime axetil) shows the second derivative spectra and the second classifier's ROC curves for the same antibiotics in Figure 3. The performances of the best classifier are summarized in Table 2.

Figure S5 (for cephalexin, ciprofloxacin, gentamicin, and sulfamethoxazole) shows the second derivative spectra and the second classifier's ROC curves for the same 


\section{Page S-3}

antibiotics in Figure S3. The performances of the best classifier are summarized in Table 2. The performance results for both RF and XGBoost, as second classifiers, are combined and summarized in Table S2. Although one classifier was rated best while the other was second in different pathogen/antibiotic combinations, each performance in the second classifier role was reasonable and comparable with the best classifier's performance.

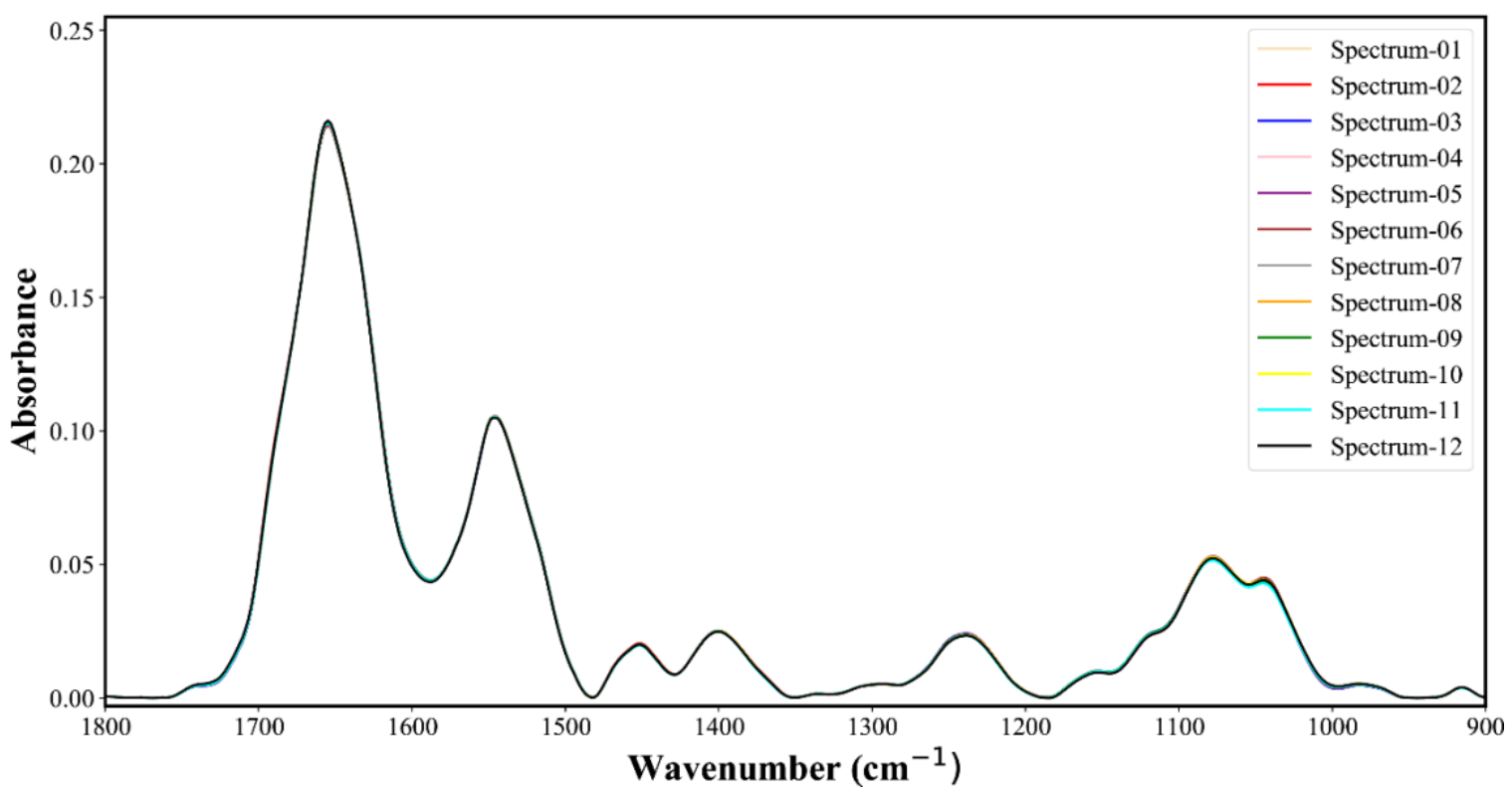

Figure S1: 12 measurements acquired from different sited of the same bacterial isolates. 
Page S-4
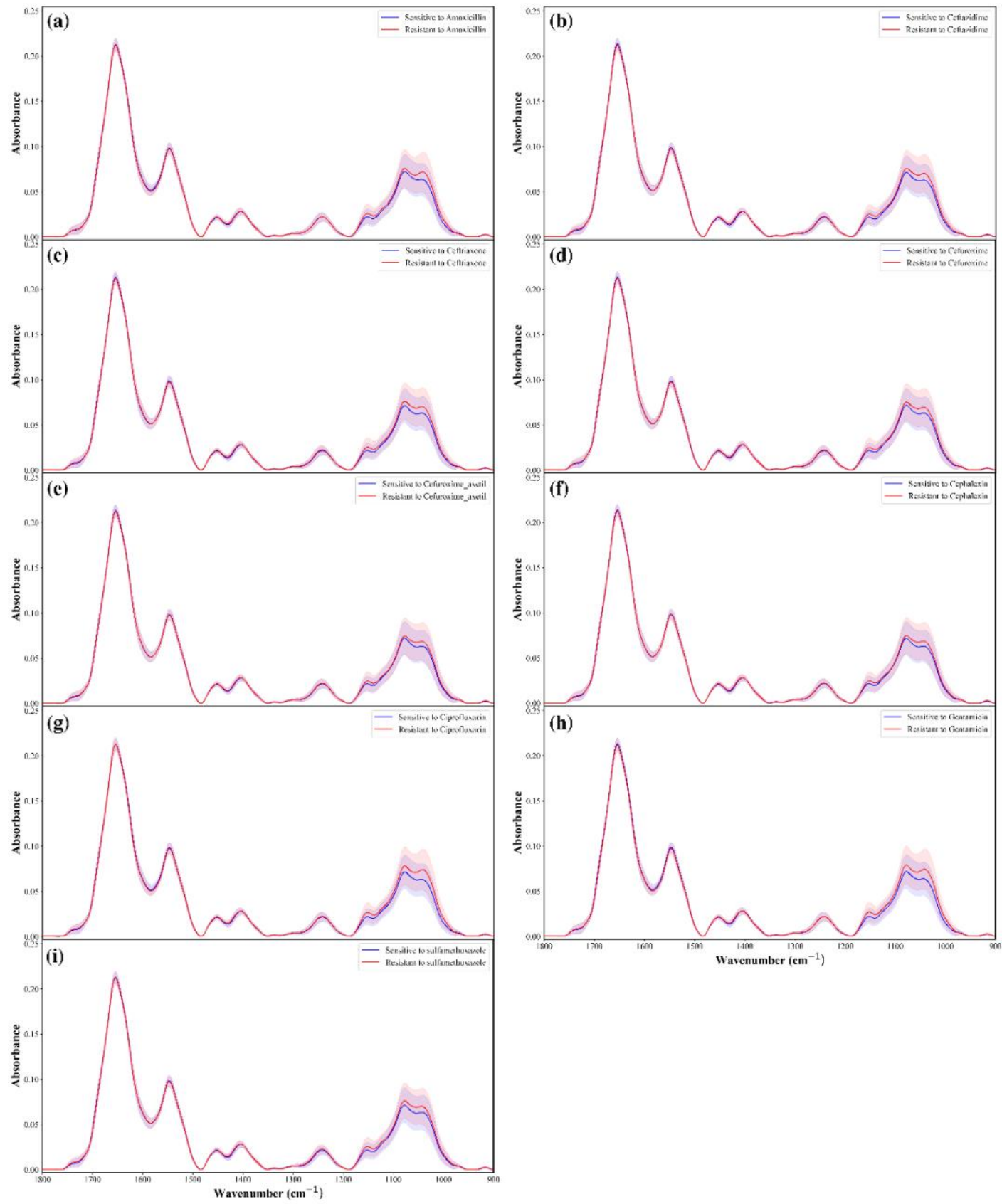

Figure S2: The average Klebsiella pneumoniae spectra grouped based on the isolate susceptibility to (a) amoxicillin, (b) ceftazidime, (c) ceftriaxone, (d) cefuroxime, (e) cefuroxime axetil, (f) cephalexin, (g) ciprofloxacin, (h) gentamicin and (i) sulfamethoxazole in the $900-1800 \mathrm{~cm}^{-1}$ wavenumber region, after pre-processing. The highlighted area represents the standard deviation of the spectra. 


\begin{tabular}{|c|c|c|c|c|}
\hline Antibiotics & Classifier & $\begin{array}{c}\text { No. of } \\
\text { selected } \\
\text { features }\end{array}$ & No. of trees & $\begin{array}{l}\text { Maximum } \\
\text { depth }\end{array}$ \\
\hline Amoxicillin & $\mathrm{RF}$ & 469 & $500-700$ & Random \\
\hline Ceftazidime & $\mathrm{RF}$ & 469 & $500-700$ & Random \\
\hline Ceftriaxone & XGBoost & 100 & $900-1100$ & $5-7$ \\
\hline Cefuroxime & $\mathrm{RF}$ & 200 & $500-700$ & Random \\
\hline Cefuroxime Axetil & XGBoost & 200 & $900-1100$ & $5-7$ \\
\hline Cephalexin & XGBoost & 100 & 900-1100 & $5-7$ \\
\hline Ciprofloxacin & XGBoost & 469 & $900-1100$ & $5-7$ \\
\hline Gentamicin & XGBoost & 200 & $900-1100$ & $5-7$ \\
\hline Sulfamethoxazole & XGBoost & 469 & 900-1100 & $5-7$ \\
\hline
\end{tabular}


Page S-6
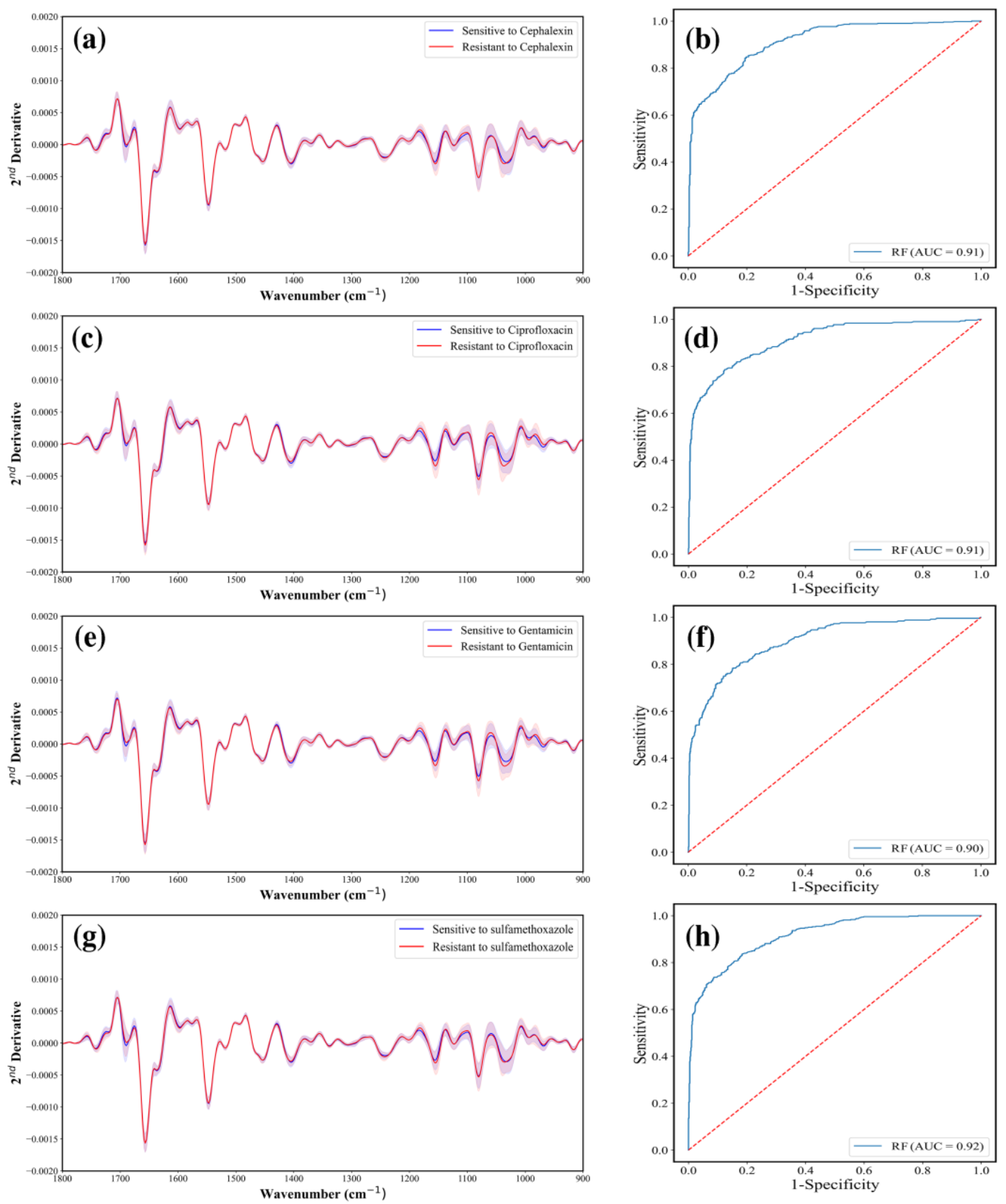

Figure S3: The average second derivative of Klebsiella pneumoniae spectra in the $1800-900 \mathrm{~cm}^{-1}$ wavenumber region, after pre-processing, grouped based on isolate susceptibility to (a) cephalexin, (c) ciprofloxacin, (e) gentamicin and (g) sulfamethoxazole. The accuracy of the classifiers was demonstrated by the ROC curve for classifying among Klebsiella pneumoniae as sensitive or resistant to the same antibiotics, respectively, is shown in Figures. (b), (d), (f) and (h). The highlighted area represents the standard deviation of the spectra. 
Page S-7
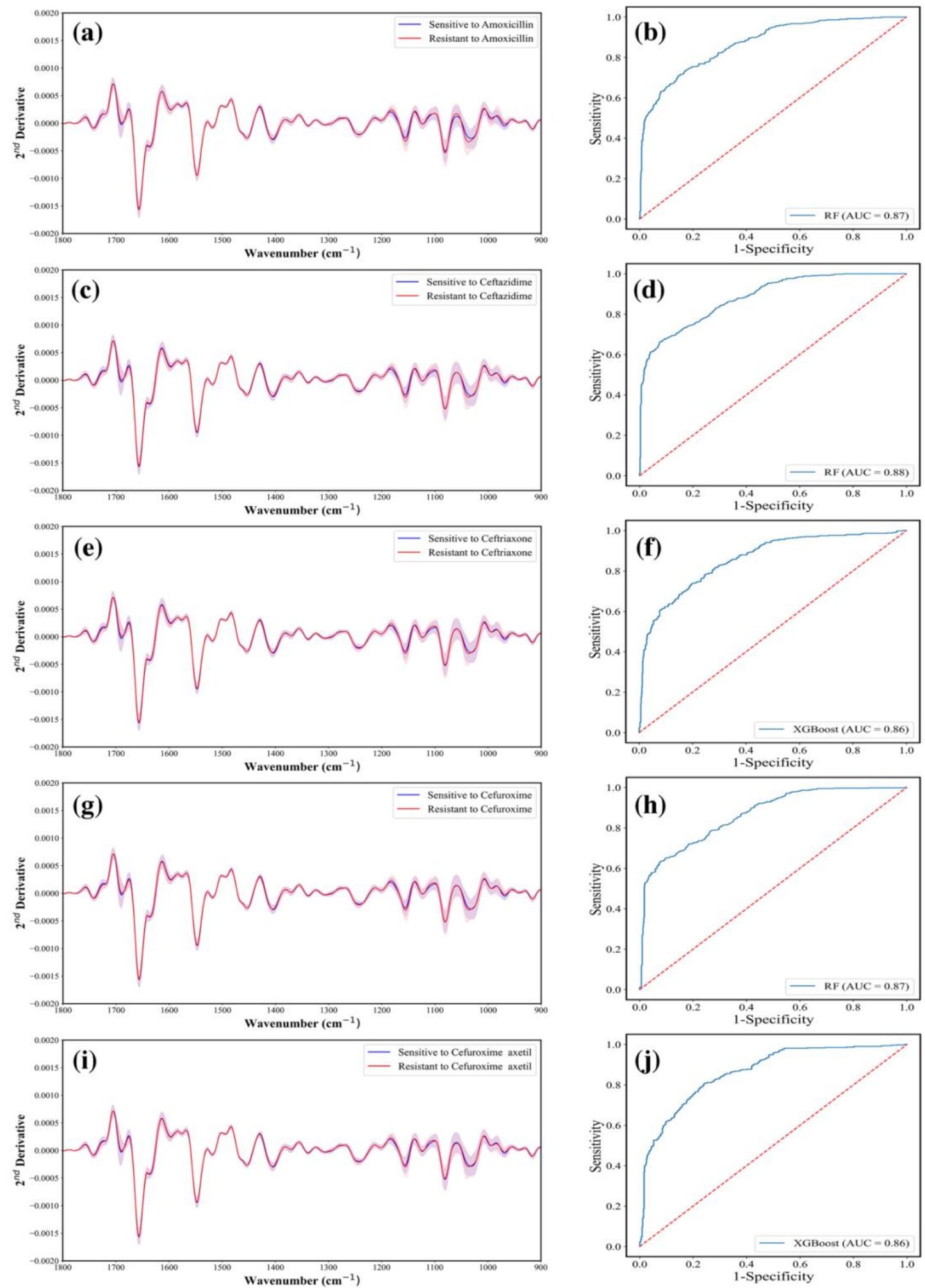

Figure S4: The average second derivative of Klebsiella pneumoniae spectra in the $900-1800 \mathrm{~cm}^{-1}$ wavenumber region, after pre-processing, grouped based on the isolate susceptibility to (a) amoxicillin, (c) ceftazidime, (e) ceftriaxone, (g) cefuroxime and (i) cefuroxime axetil. The accuracy of the classifiers was demonstrated by the ROC curve for classifying among Klebsiella pneumoniae as sensitive or resistant to the same antibiotics respectively are shown in Figures. (b), (d), (f), (h) and (j). The highlighted area represents the standard deviation of the spectra. 
Page S-8
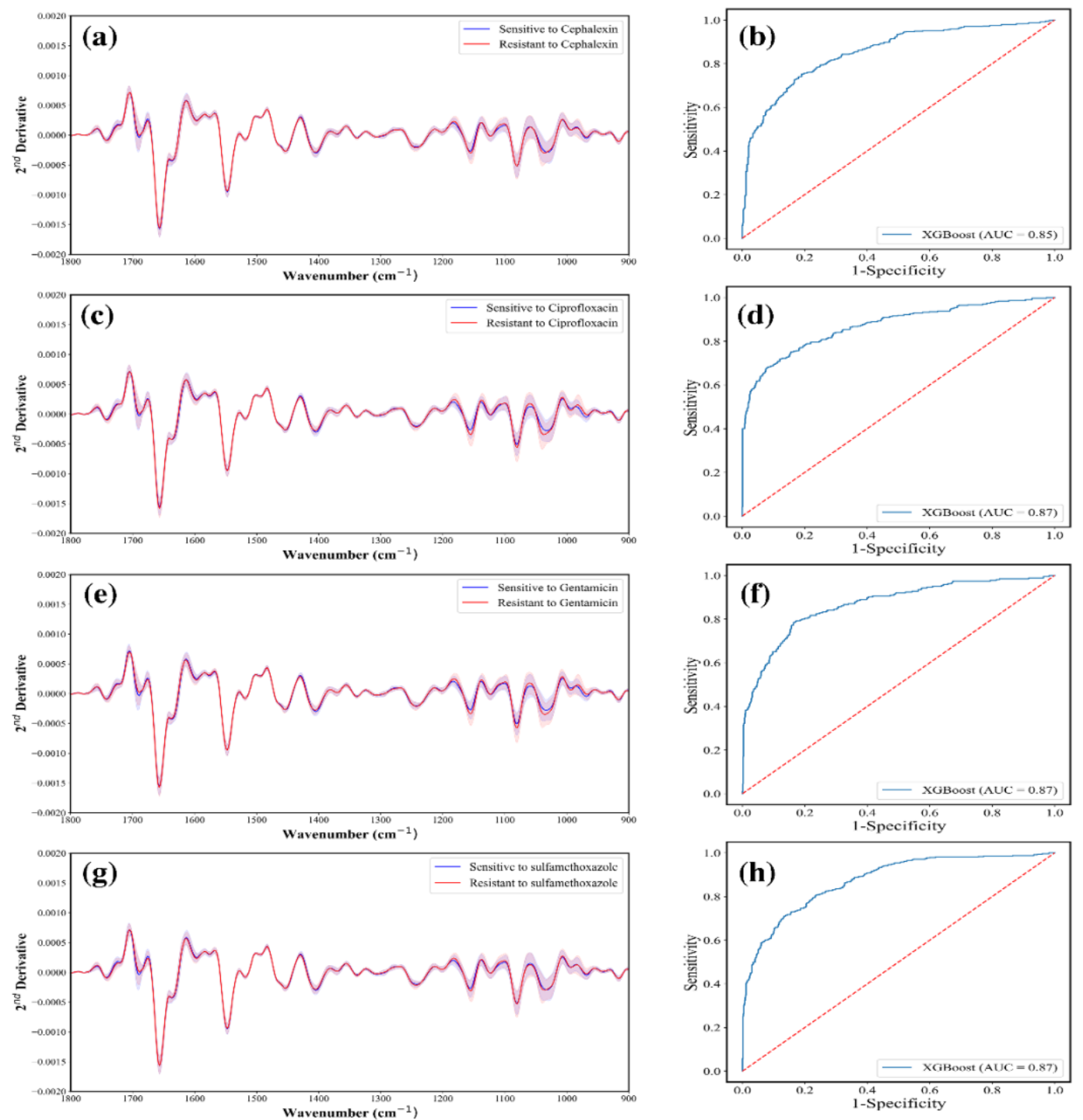

Figure S5: The average second derivative of Klebsiella pneumoniae spectra in the $900-1800 \mathrm{~cm}^{-1}$ wavenumber region, after pre-processing, grouped based on the isolate susceptibility to (a) cephalexin, (c) ciprofloxacin, (e) gentamicin and (g) sulfamethoxazole. The accuracy of the classifiers was demonstrated by the ROC curve for classifying among Klebsiella pneumoniae as sensitive or resistant to the same antibiotics respectively are shown in Figures. (b), (d), (f) and (h). The highlighted area represents the standard deviation of the spectra. 
Page S-9

\begin{tabular}{|c|c|c|c|c|c|c|c|c|}
\hline Antibiotic & $\begin{array}{c}\text { No. of } \\
\text { sensitive } \\
\text { isolates }\end{array}$ & $\begin{array}{l}\text { No. of } \\
\text { resistant } \\
\text { isolates }\end{array}$ & AUC & Accuracy & SE & SP & PPV & NPV \\
\hline Amoxicillin & 820 & 367 & 0.87 & 80 & 87 & 64 & 84 & 69 \\
\hline Ceftazidime & 645 & 521 & 0.88 & 79 & 86 & 70 & 78 & 80 \\
\hline Ceftriaxone & 679 & 507 & 0.86 & 77 & 82 & 70 & 79 & 75 \\
\hline Cefuroxime & 627 & 560 & 0.87 & 77 & 82 & 71 & 76 & 78 \\
\hline Cefuroxime Axetil & 556 & 530 & 0.86 & 78 & 78 & 78 & 79 & 77 \\
\hline Cephalexin & 588 & 512 & 0.85 & 78 & 82 & 74 & 78 & 78 \\
\hline Ciprofloxacin & 880 & 309 & 0.87 & 86 & 94 & 63 & 88 & 80 \\
\hline Gentamicin & 926 & 264 & 0.87 & 85 & 95 & 50 & 87 & 75 \\
\hline Sulfamethoxazole & 700 & 487 & 0.87 & 80 & 86 & 71 & 81 & 78 \\
\hline
\end{tabular}

